(e.g., schedules) and offering injury prevention education (e.g., theory, practical skills).

Conclusions This study provided insight into how injury prevention is shaped in practice, identified critical factors and motives for injury prevention, and mapped recommendations for its improvement from the target population. The findings support the development of context-driven preventive strategies in the PETE population.

\section{COMPREHENSIVE ASSESSMENT OF RISK FACTORS AND CONSTANT LOAD MONITORING PREVENT OVERUSE INJURIES OF EXTENSOR MECHANISM IN ELITE VOLLEYBALL PLAYERS} University Gülhane Medical Faculty, Department of Sports Medicine, Ankara, Turkey; ${ }^{2}$ Halkbank Man Volleyball Team, Ankara, Turkey

\subsection{6/bjsports-2021-IOC.198}

Background Overuse injuries of extensor apparatus are common and debilitating in elite volleyball. Addressing the internal risk factors and monitoring the training load are key for injury prevention.

Objective To highlight the effectiveness of preventive measures on an elite male volleyball team in 2 consecutive seasons.

Design Overuse injury incidence of extensor mechanism, taken preventive measures and assessment strategies of injury risk factors in an elite level man volleyball team are investigated retrospectively using records of the team.

Setting Male volleyball team competing in elite division.

Participants The players of Halkbank Volleyball Team during the 2019-2020 and 2020-2021 seasons except liberos.

Assessment of Risk Factors Functional movement system test and Y-balance test were conducted and 1-RMs were assessed after pre-participation evaluation. Linear periodization was used during the pre-season preparation period. Individual strength-mobility deficits and asymmetries of players were targeted with supervised exercise sessions in addition to regular fitness trainings. The players' total load on extensor apparatus during trainings and games were monitored with the G-Vert, a commercially available wearable device measuring vertical displacement and jump count. The target values as maximum tolerable jump counts were established for each position and for each player who had previous extensor apparatus injury. The training volume was adjusted when the target value is achieved.

Main Outcome Measurements The primary study outcome measure was the time loss due to extensor mechanism injuries.

Results A comprehensive approach to prevent overuse injuries of the extensor mechanism yielded excellent results in an elite division volleyball team. There was no time loss due to overuse injuries of extensor apparatus despite the heavy training and the game load.

Conclusions Reducing and monitoring the internal risk factors combined with constant monitorization and establishment of a target jump load in volleyball prove to be useful. Considering the technical difficulties to conduct a high-quality randomized research on elite athletes, comprehensive preventive measures applied for two years show promising results.

\section{WHO'S KEEPING SCORE? THE EFFECT OF A SCORE DIFFERENTIAL BASED RUNNING TIME RULE ON HEAD IMPACT RATES IN CANADIAN HIGH SCHOOL TACKLE FOOTBALL}

${ }^{1,3,9}$ M Patrick Pankow, ${ }^{1,3,9}$ Reid A Syrydiuk, ${ }^{1,3,9}$ Ash T Kolstad, 'Sagar Grewal, ${ }^{1,3}$ Christian A Clermont, ${ }^{8}$ Christopher R Dennison, ${ }^{1,3,6,7,9}$ Brent E Hagel, ${ }^{2}$ Martin Mrazik, $1,3,4,5,6,7,9$ Carolyn A Emery. 'Sport Injury Prevention Research Centre, Faculty of Kinesiology - University of Calgary, Calgary, Canada; ${ }^{2}$ Faculty of Education - University of Alberta, Edmonton, Canada; ${ }^{3}$ Alberta Children's Hospital Research Institute, Calgary, Canada; ${ }^{4}$ Hotchkiss Brain Institute - University of Calgary, Calgary, Canada; ${ }^{5}$ Evidence Sport and Spinal Therapy, Calgary, Canada; ${ }^{6}$ Departments of Paediatrics and Community Health Sciences, Cumming School of Medicine - University of Calgary, Calgary, Canada; ${ }^{7}$ O'Brien Institute for Public Health, Cumming School of Medicine - University of Calgary, Calgary, Canada; ${ }^{8}$ Department of Mechanical Engineering - University of Victoria, Victoria, Canada; ${ }^{9}$ Integrated Concussion Research Program - University of Calgary, Calgary, Canada

\subsection{6/bjsports-2021-IOC.199}

Background Due to postulated associated long-term health issues in athletes, concussions and head impacts are of concern in tackle football. Football Canada mandated a game clock running-time rule (RTR) in the event of a second-half 35 point difference in games, citing player safety as the main rationale.

Objective To examine the effectiveness of RTR on reducing game-related head impact rates in Canadian high school football using video analysis.

Design Cross-sectional.

Setting Calgary, Canada.

Participants Players on two junior division high school teams (ages 15-16) in Calgary, Alberta were included. Fourteen games from the 2019 season (Team A: $n=8$, Team B: $n=6$ ) were videotaped for analyses.

Assessment of Risk Factors Traditionally, the clock stops between plays until the referee signals for the clock to resume. With RTR the clock continues (except during exceptional circumstances such as injury, scores, or timeouts) in the event of a point differential of 35 points or greater in the second half of a game.

Main Outcome Measurements Head impacts were reported as incidence rates (IR) [\# head impacts/100 player-game-minutes (PGM) (95\% confidence intervals $(95 \% \mathrm{CI})]$. Incidence rate ratios (IRR), offset for PGM, adjusted for game outcome (e.g., win, loss) and clustering by team game were used to compare score differential in games with and without running-time ( $\geq 35$ points vs. $<35$ points) by team unit (e.g., offense, defense).

Results RTR games yielded 24\% fewer plays than non-RTR games (IRR: 0.76, 95\% CI: 0.68, 0.84). Head impact IR in RTR games were lower than non-RTR games for offensive units (IRR:0.80; 95\% CI:0.68, 0.95) and defensive units (IRR:0.76; 95\% CI:0.59, 0.99). There were no differences in special teams units.

Conclusions RTR reduced game-related head impact IRs in this cohort for both offensive and defensive units. Sport governing bodies should consider the potential effect of RTR on injury and concussion rates at the youth level. 